\title{
Fast Network Oscillations in the Newborn Rat Hippocampus In Vitro
}

\author{
J. Matias Palva, ${ }^{1}$ Karri Lamsa, ${ }^{1}$ Sari E. Lauri, ${ }^{1,2}$ Heikki Rauvala, ${ }^{2}$ Kai Kaila, ${ }^{1}$ and Tomi Taira ${ }^{1}$ \\ ${ }^{1}$ Department of Biosciences, Division of Animal Physiology, and 2Laboratory of Molecular Neurobiology, Institute of \\ Biotechnology, and Department of Biosciences, University of Helsinki, FIN-00014 Finland
}

Spontaneous neural activity is crucial for the formation of the intricate patterns of cortical connectivity during development. In particular, temporal correlations in presynaptic and postsynaptic activity have been hypothesized to be a critical determinant in the selection of neurons that are to become wired together. To date, however, temporally correlated activity in the neonatal brain has been believed to take place with a precision of tens of milliseconds to seconds.

Here we describe a novel type of a fast network oscillation associated with millisecond synchronization of pyramidal cell firing in newborn rat hippocampus in vitro. Individual pyramidal neurons fired mainly at lower gamma frequencies $(20-40 \mathrm{~Hz})$ but were synchronized into a high-frequency $(100-400 \mathrm{~Hz})$ population oscillation that was reflected in field potential spikes and intracellular AMPA-kainate receptor-mediated currents. The high-frequency population oscillation was patterned by a gamma-frequency modulatory oscillation. The gamma modulation was imposed by GABAergic currents, which exerted an inhibitory action on pyramidal neurons. Patterned activity based on GABAergic inhibition and glutamatergic excitation thus occurs already in newborn hippocampus. The network oscillations described here may be a mechanism for selective coincidence detection with a millisecond range temporal precision to shape the patterns of connectivity within the emerging hippocampal synaptic circuitry.

Key words: network oscillations; synchronization; neonate; coincidence detection; $\mathrm{GABA}_{A}$; inhibition; plasticity
Neural circuits are constructed to a great degree of elaboration before the onset of experience-dependent plasticity. Spontaneous neural activity plays a central role in shaping the developing neural networks (Katz 1993; Katz and Shatz, 1996). Recurrent bursts of network activity are a general feature of the developing CNS in vertebrates (O'Donovan, 1999). In the newborn rat hippocampus, spontaneous activity is characterized by population bursts suggested to result from synergistic excitation mediated by $\mathrm{GABA}_{\mathrm{A}}$ and NMDA receptors (Leinekugel et al., 1997; Strata et al., 1997). This activity is tightly linked to slow, globally synchronous $\mathrm{Ca}^{2+}$ waves suggested to control the formation of new synapses in the developing hippocampus (Durand et al., 1996; Ben-Ari et al., 1997; Garaschuk et al., 1998). It has been suggested that a critical factor for a given set of neurons to become functionally connected is the temporal coincidence of their activity (Katz, 1993). Consequently, the width of the temporal integration window defines the minimum rate at which populations can be dissociated; a narrower integration window would allow for a larger number of independent coincidences in a given time.

In the adult brain, gamma- $(20-100 \mathrm{~Hz})$ (Gray et al., 1989; Bragin et al., 1995; König et al., 1995; Laurent, 1997) and highfrequency (100-200 Hz) network oscillations (Ylinen et al., 1995) appear to be central for the timing and coordination of neural

\footnotetext{
Received April 22, 1999 ; revised Nov. 11, 1999; accepted Nov. 11, 1999.

This work was supported by the Academy of Finland, the Sigrid Juselius Foundation, and the Oskar Öflund foundation. We are grateful to Klaus LinkenkaerHansen and Satu Palva for helpful discussions.

J.P. and K.L. contributed equally to this work.

Correspondence should be addressed to Tomi Taira, Department of Biosciences, Division of Animal Physiology, P.O. Box 17, FIN-00014 University of Helsinki, Finland. E-mail: tomi.taira@helsinki.fi.

Copyright (C) 2000 Society for Neuroscience $\quad 0270-6474 / 00 / 201170-09 \$ 15.00 / 0$
}

activity (Ritz and Sejnowski, 1997). Furthermore, synchronized afferent firing is required for the induction of dendritic backpropagating action potentials implicated in associative synaptic potentiation (Markram et al., 1997; Traub et al., 1998). The generation of gamma- and high-frequency oscillations in the mature hippocampus relies on AMPA receptor-mediated excitation as well as hyperpolarizing $\mathrm{GABA}_{\mathrm{A}}$ receptor-mediated inhibition and GABAergic interneuron networks (Lytton and Sejnowski, 1991; Cobb et al., 1995; Whittington et al., 1995; Ylinen et al., 1995; Fisahn et al., 1998). Thus, because of the alleged quiescence of AMPA synapses (Durand et al., 1996), the depolarizing nature of $\mathrm{GABA}_{\mathrm{A}}$ responses (Ben-Ari et al., 1989; Rivera et al., 1999), and the suggested lability of functional $\mathrm{GABA}_{\mathrm{A}}$ receptor-mediated inhibition in the rat hippocampal pyramidal cells during the first postnatal week (Luhmann and Prince, 1991; Ben-Ari et al., 1997; Leinekugel et al., 1997), it has been assumed that developing hippocampal networks lack the critical synaptic circuitry required for fast population oscillations mediating sharp synchronization (Traub et al., 1998).

We have assessed the temporal precision of correlated activity in developing hippocampal networks. Here we show that spontaneous fast population oscillations, which allow for and coordinate precise synchronization of neural activity, are indeed found in the hippocampus already during the first postnatal days.

\section{MATERIALS AND METHODS}

Electrophysiology. Six hundred micrometer hippocampal transverse slices were prepared from postnatal day 3 (P3)-P6 Wistar rats using a McIlwain tissue chopper. The day of birth is referred to as $\mathrm{P} 0$. The slices were allowed to recover at room temperature $\left(20-22^{\circ} \mathrm{C}\right)$ for at least $1 \mathrm{hr}$ in oxygenated $\left(95 \% \mathrm{O}_{2}\right.$ and $\left.5 \% \mathrm{CO}_{2}\right)$ physiological solution containing (in mm) $\mathrm{NaCl} 124, \mathrm{KCl} 3.0, \mathrm{CaCl}_{2} 1.5, \mathrm{NaHCO}_{3} 25, \mathrm{NaH}_{2} \mathrm{PO}_{4} 1.1, \mathrm{MgSO}_{4}$ 1.3, and D-glucose 11, $\mathrm{pH}$ 7.4. Experiments were performed in a submerge-type chamber at $32^{\circ} \mathrm{C}$ where slices were placed on a mesh and 

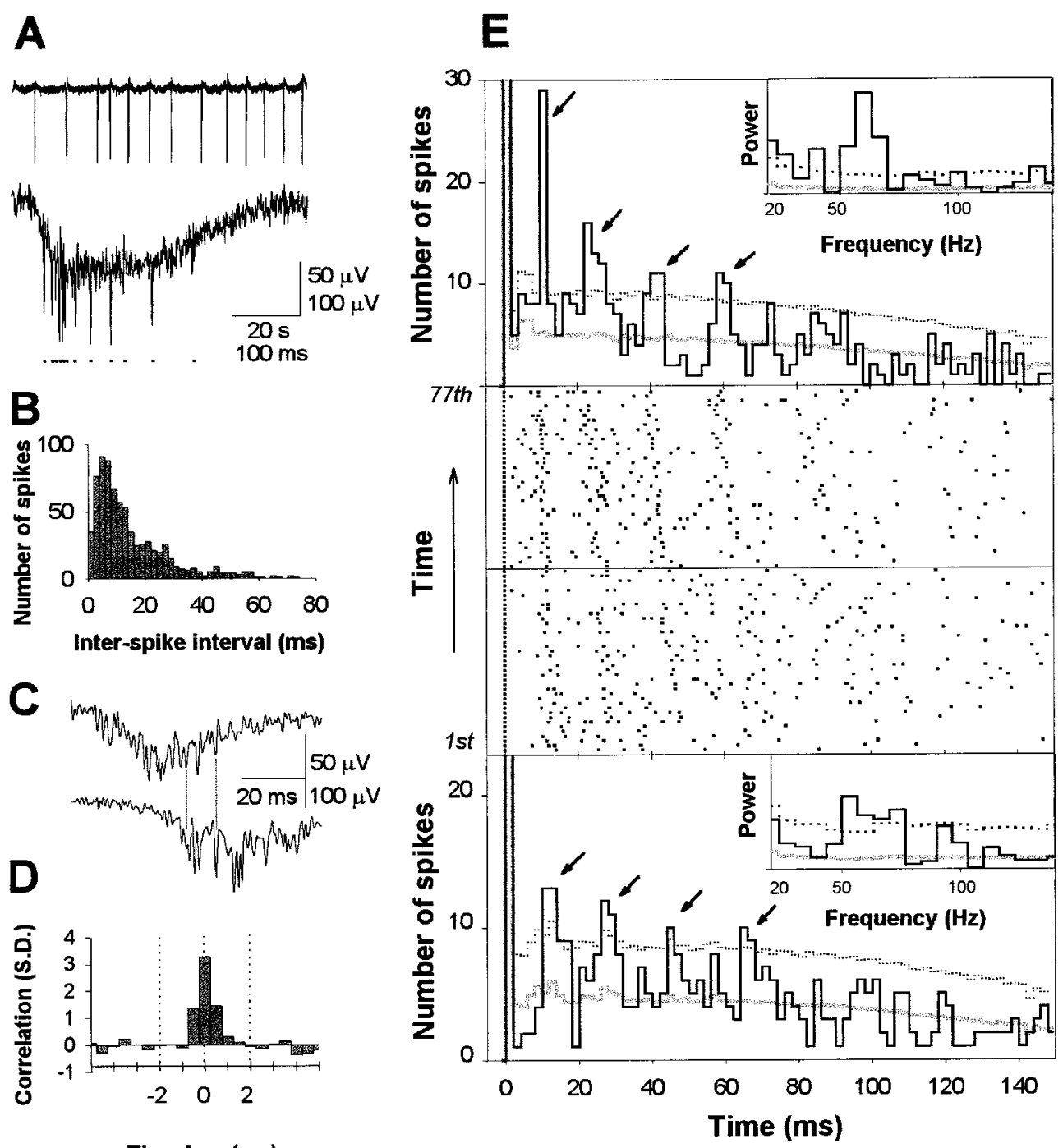

Time Lag (ms)

Figure 1. Millisecond range synchronization and gamma-frequency modulation of the field potential spikes. $A$, Population activity is seen in filtered (20 $\mathrm{Hz}$ low-pass) field potential recordings as a continuous stream of negative deflections (top trace). Each negative deflection is associated with a burst of field potential spikes (bottom trace) (low-pass filtered at $700 \mathrm{~Hz}$ ). The field potential spikes are marked with dots below the trace. $B$, The distribution of interspike intervals in this experiment ( 675 spikes in 77 bursts) $C$, A burst of population spikes recorded simultaneously with two extracellular electrodes separated by $100 \mu \mathrm{m}$. $D$, An averaged cross-correlogram of 149 pairs of population bursts in three recordings showing that the synchronized field potential spikes coincide with a jitter of $<1 \mathrm{msec}$. The correlation is before averaging expressed as the number of baseline SDs between the correlogram and baseline mean. E, Middle panel, The occurrence of field potential spikes (marked with dots) after the first spike of the burst is rhythmically patterned. One horizontal strip of dots represents the spikes of one burst. All 77 bursts in this experiment are shown starting from the bottom. Note how the vertical columns of spikes are systematically tilted to the left, demonstrating the time-varying nature of the modulation. Top and bottom panels, Histograms of spike occurrence modulation. The first half of the experiment is pooled to the lower histogram, and the second half to the upper. The dotted lines represent the $95 \%$ confidence limits (mean +2 SDs of shuffled histograms). Gray lines indicate the mean of the shuffled histograms (see Materials and Methods). The arrows show the four significant peaks in both histograms. Insets, Power spectra of the corresponding spike occurrence histograms showing highly significant gamma-frequency oscillations. The dotted lines denote the $99 \%$ confidence limits (mean +3 SDs of power spectra computed for shuffled histograms). The mean of shuffled data are shown by the gray lines.

superfused with the physiological solution at a rate of $3-4 \mathrm{ml} / \mathrm{min}$. Field potential recordings were made with conventional NaCl-filled $(150 \mathrm{~mm})$ glass capillary microelectrodes $(5-10 \mathrm{M} \Omega)$. Whole-cell recordings were obtained from cells in the CA3 region. The filling solution used in whole-cell micro pipettes (4-6 M $\Omega$ ) was (in $\mathrm{mM}$ ): K-gluconate 140, $\mathrm{CaCl}_{2}$ 3, EGTA 6, HEPES 10, and Mg-ATP 2, pH 7.30.

Data analysis. The signals were low-pass filtered at 700 or at $1100 \mathrm{~Hz}$, tape recorded with a TEAC SR-31 recorder, and digitized off-line at 2 or $4 \mathrm{kHz}$ with a National Instruments AT-MIO-16-E-2 analog-to-digital board and LabView software (National Instruments). Decay time constants of fast synaptic currents were estimated by a least-squares exponential fit.

The field potential spike and AMPA-kainate current peak latencies were determined from high-pass filtered $(20 \mathrm{~Hz})$ spontaneous events with a peak detection algorithm (LabView) and verified visually. We analyzed only those field potential recordings in which, on average, more than four spikes occurred during burst, and a total of more than 200 spikes were detected. Spikes and current peaks with an amplitude greater than three times the preburst SD were accepted. We examined the temporal patterning in bursts of field potential spikes with two approaches. First, we computed histograms of spike occurrence after the first spike in the burst by pooling the spike latencies from a large number $(30+)$ of consecutive bursts (bin width 1, 2, or $4 \mathrm{msec}$ ). Confidence limits were estimated by computing the mean and SD of shuffled histograms. The shuffling (random mixing of interspike intervals) was computed within each burst before the computation of the histogram. At least 1000 randomizations 


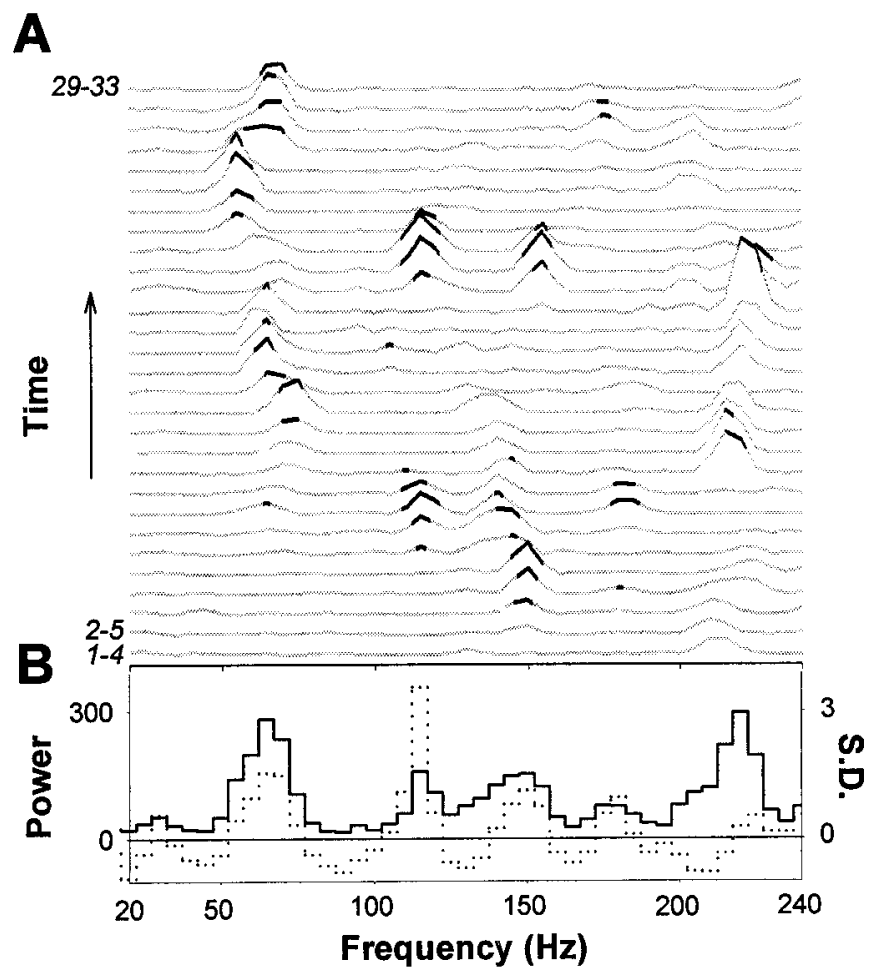

Figure 2. Transient gamma- and high-frequency oscillations in field potential spike bursts. A, Power spectra of all 29 cumulative ACHs in this experiment. Four consecutive spike bursts were taken into each $\mathrm{ACH}$, and this window of four was shifted one at a time. Black parts of the power spectra are above the $99 \%$ confidence limit. $B$, Averaging the power spectra of 29 ACHs (33 spike bursts) abolishes significant oscillations. Black line, Mean of the power spectra in A; dotted line, mean of the normalized power spectra. The spectra were normalized by subtracting the mean of shuffled data and dividing with the SD. Significant oscillations in most of the power spectra thus fall below the significance level when averaged.

were computed for the estimation of mean and SD for each histogram bin. Peaks exceeding the mean $+2 \mathrm{SD}$ were accepted as significant. The presence of rhythmic spike occurrence modulation was quantified by computing power spectra of the histograms. Confidence limits were estimated from the power spectra of shuffled histograms; peaks exceeding mean $+3 \mathrm{SD}$ were considered significant. We studied finer temporal patterning with autocorrelation histograms (ACHs). Single bursts had generally too few spikes for $\mathrm{ACH}$ analysis, and we thus computed cumulative ACHs of typically four (but also up to 10) successive spike bursts. The window of four or more bursts was slid over all bursts in the experiment with steps of one or two bursts. Periodicity in each cumulative $\mathrm{ACH}$ was quantified with its power spectrum. Confidence limits for the power spectra were estimated by computing the cumulative ACHs for pseudorandom spike bursts. The randomized bursts had an equal number of spikes and an identical interspike interval distribution as the original bursts. At least 300 randomizations were taken for the mean and SD of shuffled power spectra. We accepted peaks exceeding the mean $+3 \mathrm{SD}$ as significant. Because the oscillation frequency varied not only across but also within bursts, and the temporal dispersion of the spikes was more pronounced in the late parts of the bursts, the $\mathrm{ACH}$ analyses were computed for the first $100 \mathrm{msec}$ of each burst.

For the time-frequency analysis, the signal was convoluted with a Gabor wavelet: $h(t, f)=\exp \left(-x^{2} / 2+i m x\right), x=2 \pi f t / m$, where time and frequency are denoted with $t$ and $f, m=9$, and $i$ is the imaginary unit. Modulus of the complex valued outcome represents the amplitude of the signal at a narrow frequency band as a function of time (Sinkkonen et al., 1995). To cover the desired range of frequencies, the center frequency $f$ of the wavelet was varied in small steps $(1-5 \mathrm{~Hz})$, and the convolution was performed 50-200 times. The squared time frequency (TF) representations were averaged using as a trigger either the current maximum $\left(\mathrm{GABA}_{\mathrm{A}}\right.$ currents) or the first field potential spike of a simultaneously recorded field potential event (AMPA-kainate currents), or, when the field potential was not recorded, the first AMPA-kainate current peak of each burst. TF analysis of amplitude modulation of a high-frequency oscillation by a lower-frequency oscillation was performed by first averaging the amplitude envelopes within the high-frequency band (computed with $m=6$ ) and then computing a new (squared) TF representation for the averaged amplitude envelope. The high-frequency bands were selected around prominent peaks in the averaged TF representation of the bursts. As a criterion of significance for the TF representations and $\mathrm{TF}$ analyses of amplitude modulation, we required the TF peak energy to exceed the mean +3 SDs of the 400 msec preceding the burst.

The correlation between field potential spikes and GABAergic currents was analyzed by averaging the GABAergic current around each field potential spike in the bursts. Before averaging the currents, leastsquares fitted third order polynomials were subtracted to remove the underlying slow shifts, and the currents were normalized to vary between -1 and 1 .

The analysis software, programmed in the LabView environment, is available upon request from J.M.P. (E-mail: jpalva@cc.hut.fi).

\section{RESULTS}

\section{Synchronization and oscillatory patterning of activity in populations of pyramidal cells}

We first addressed the presence of correlated activity in populations of pyramidal neurons. Bursts of activity, occurring at interburst intervals of $18 \pm 3 \mathrm{sec}$ (mean \pm SEM), were seen in field potential recordings from the CA3 pyramidal cell layer in 33 of 42 slices studied. In 27 of 33 recordings we found one or more field potential spikes to accompany the slow negative deflections in field potential (Fig. $1 A$ ). The field potential spikes had a mean amplitude of $90 \pm 18 \mu \mathrm{V}$ and an average width of $2.5 \pm 0.5 \mathrm{msec}$ at the base. The distributions of interspike intervals were typically broad, peaking between 5 and $20 \mathrm{msec}$ (Fig. $1 B$ ). To confirm that the spikes reflected population activity and not the firing of single neurons, we also recorded simultaneously with two extracellular electrodes separated by $100 \mu \mathrm{m}$ at the tip (149 bursts in three slices). It is unlikely that the firing of a single neuron could be picked up by both electrodes across such a distance (Draguhn et al., 1998). Indeed, in all recordings, some of the spikes were found to occur simultaneously (Fig. 1C). Cross-correlation analyses showed a sharp peak at 0 msec mean time lag confirming the presence of spikes synchronized with millisecond precision (Fig. 1D).

To study the coarse temporal structure of the spike bursts, we computed histograms of spike occurrence after the first spike of each burst. The confidence limits for the histograms were estimated by shuffling the interspike intervals within each burst and computing the mean and SD of the shuffled histograms (see Materials and Methods). We considered peaks exceeding the mean \pm 2 SDs (i.e., $95 \%$ confidence limit) as significant. In eight of nine recordings, two or more peaks exceeded the significance level. The peaks occurred regularly with interpeak intervals in the range of $10-20 \mathrm{msec}$ (Fig. 1D). Periodic modulation in the histograms was quantified with power spectra. Confidence limits were estimated by computing the power spectra for shuffled histograms (see Materials and Methods); a peak was considered significant if it exceeded the mean \pm 3 SDs (the $99 \%$ confidence limit). In eight of nine power spectra, one or more peaks exceeded the significance level. In seven of eight recordings, the peaks were in the gamma frequency band (mean $76 \pm 7 \mathrm{~Hz}$ ) (Fig. $1 E$ ).

We then investigated the finer temporal structure of the spike bursts with power spectra computed from ACHs. Because the number of spikes in individual bursts was generally inadequate for $\mathrm{ACH}$ analysis, we pooled data from four or more consecutive bursts. The confidence limits for the power spectra of these 


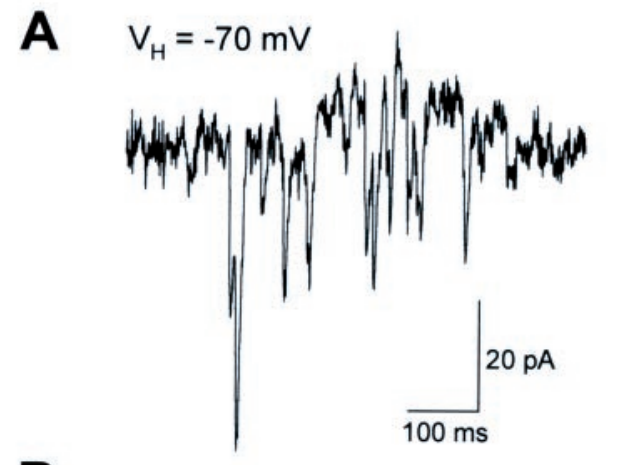

B

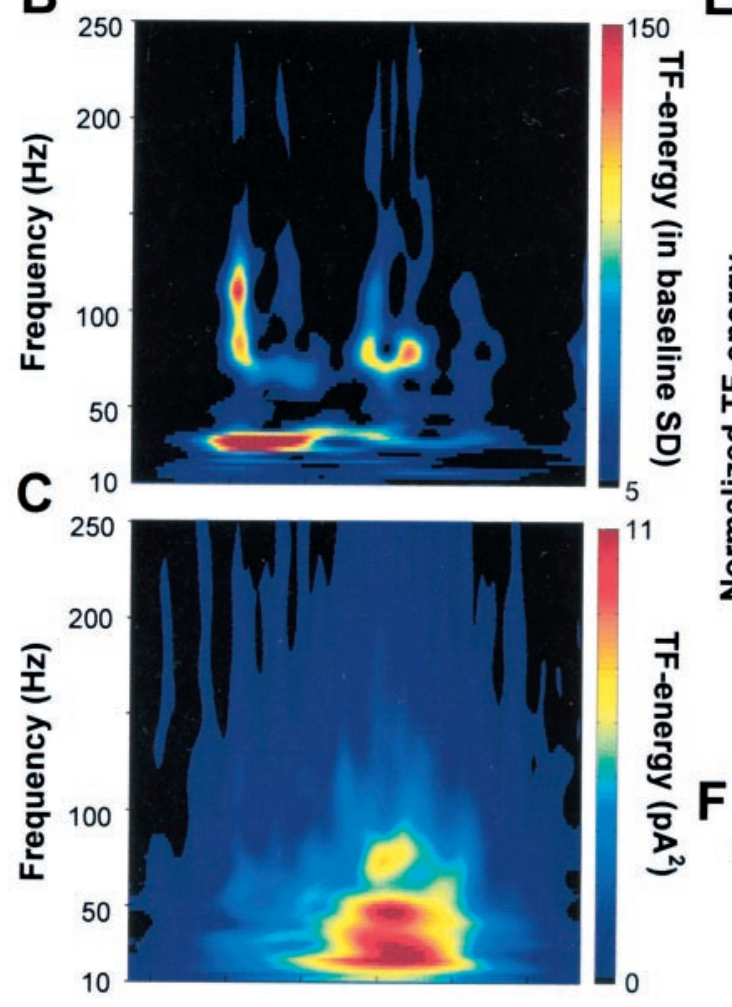

Time

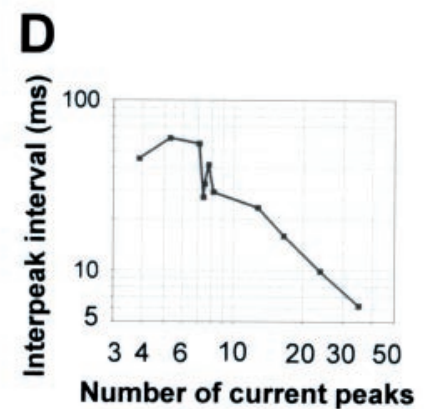

E

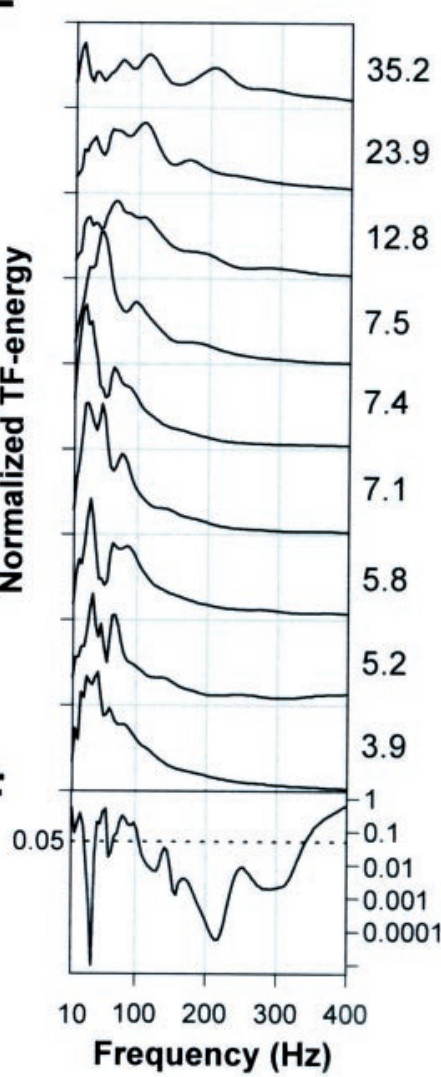

Figure 3. During spontaneous network events, AMPA-kainate receptor-mediated currents show oscillations at gamma- and high-frequencies. $A, \mathrm{~A}$ spontaneous event in a P5 pyramidal neuron clamped at $-70 \mathrm{mV}$ showing the AMPA-kainate receptormediated glutamatergic currents. $B, \mathrm{~A}$ TF representation of the glutamatergic currents in $A$. Time runs along the $x$-axis and frequency along the $y$-axis. The time scale is the same as in the current trace above the TF representation. The amplitude is coded in color so that red color corresponds to higher amplitude (see Materials and Methods for details). The high-frequency components are related to the current clusters occurring at gamma frequencies. $C$, An averaged TF representation of 103 consecutive spontaneous bursts of AMPA-kainate currents displaying the major peak in the lower $(20-40 \mathrm{~Hz})$ and a subsidiary peak in the upper $(50-80 \mathrm{~Hz})$ gamma-frequency band. $D$, The mean interpeak interval was dependent on the mean number of AMPAkainate current peaks in bursts $(p<0.005$; one-way ANOVA). E, Frequency distributions of the AMPA-kainate receptormediated current bursts in nine neurons. The distributions were obtained by averaging the TF representations of all current bursts (33-103 averages) in a single recording and then averaging 25-100 msec around the major peak. The distributions are normalized to have unit area. The mean number of current peaks during a population burst is given right to the distributions. $F$, Significance level as a function of frequency (one-way ANOVA). A below average amount of AMPA-kainate inputs per burst was associated with pronounced gammafrequency oscillations, whereas large numbers of AMPA-kainate inputs/burst were found in neurons showing prominent highfrequency oscillations. cumulative ACHs were computed again with randomized data (see Materials and Methods). In all nine recordings, significant $(>$ mean $+3 \mathrm{SD})$ gamma and high-frequency peaks were found (361 peaks in 450 power spectra; $0.8 \pm 0.1$ peaks/spectrum). The oscillations, however, were highly transient and had a variable frequency content during the course of the experiment (Fig. 2A); significant peaks were averaged out when all bursts within an experiment (ranging from 33 to 109) were pooled together (Fig. 2B).

Two explanations for the apparent discrepancy between the prominent oscillations in individual bursts and the relatively smooth distribution of frequencies in pooled data can be given. First, the field potential spikes may occur randomly at gamma and high frequencies without a dominant population frequency other than the gamma-frequency spike occurrence modulation. Alternatively, a high-frequency population oscillation may underlie the field potential spike bursts but is spatially distributed so that an extracellular electrode picks up only parts of the oscillation (see below).

\section{High-frequency pyramidal cell population oscillation is reflected in AMPA-kainate receptor-mediated currents}

To gain insight into the origin of the extracellular oscillatory components, we made intracellular recordings from CA3 and CA1 neurons simultaneously with the extracellular measurement. The neurons were recorded at a distance of 100-200 $\mu \mathrm{m}$ from the extracellular electrode and voltage-clamped near $\mathrm{E}_{\mathrm{GABA}-\mathrm{A}}$ (less than $-70 \mathrm{mV}$; electrode $\left[\mathrm{Cl}^{-}\right]=3 \mathrm{~mm}$, calculated $\mathrm{E}_{\mathrm{Cl}-}<-100$ $\mathrm{mV}$, for the deviation of $\mathrm{E}_{\mathrm{GABA}-\mathrm{A}}$ from a calculated $\mathrm{E}_{\mathrm{Cl}-}$; Kaila 1994). During the field potential bursts 2-49 sharp inwardly directed current peaks (mean $12 \pm 3$ peaks) were detected in the whole-cell recording. The current peaks had a mean decay time constant of $2.7 \pm 0.3 \mathrm{msec}$ and reversal potential positive to $0 \mathrm{mV}$ (Fig. $3 A ; n=11$ cells), indicating that they were mediated by the AMPA-kainate-type glutamate receptors (Hestrin et al., 1990). Bath application of the AMPA-kainate-type glutamate receptor antagonist 6-nitro-7-sulfamoylbenzo(f)quinoxaline-2,3-dione (20 

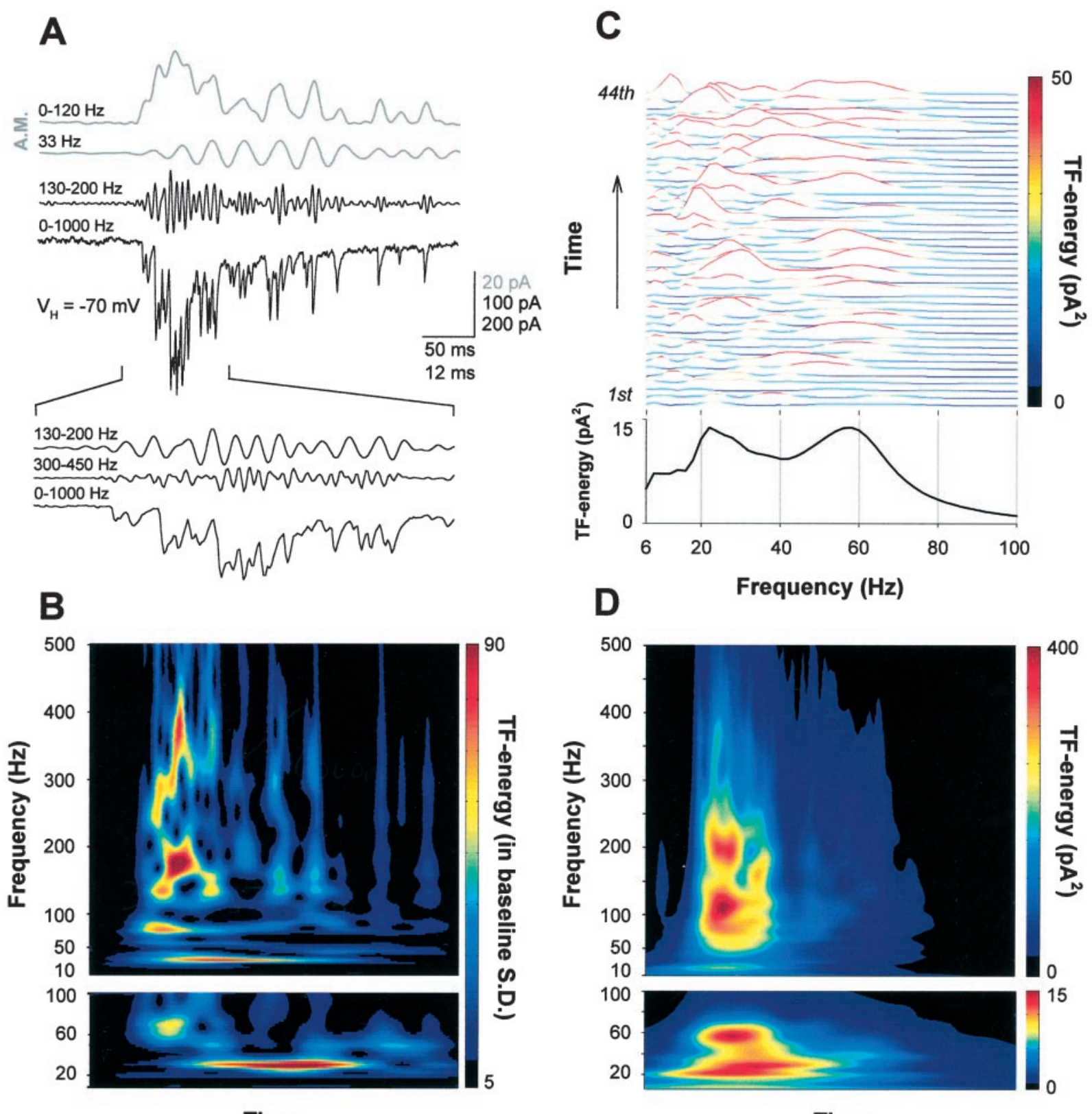

Time

Time

Figure 4. High-frequency AMPA-kainate currents are amplitude modulated at gamma frequencies. A, Two top traces ( gray), amplitude envelope of the $100-450 \mathrm{~Hz}$ frequency band (filtered as indicated in the figure). Two middle traces, The band-pass filtered and the original high-frequency burst of AMPA-kainate currents. Note how the high-frequency oscillation is amplitude modulated at $\sim 30 \mathrm{~Hz}$. Three lowest traces, The first 100 msec of the AMPA-kainate current burst is expanded to show the high-frequency oscillations around 180 and $330 \mathrm{~Hz}$ (see $B$ ). $B$, Top panel, A TF representation of the original current trace in $A$. The time scale is the same as in the four top current traces. Bottom panel, A TF representation of the amplitude envelope (highest current trace). C, Top panel, Frequency distributions of the amplitude modulation for all 44 bursts in this experiment. The amplitude modulation was computed for high-frequency oscillations around $210 \mathrm{~Hz}(160-260 \mathrm{~Hz}$, see $D)$. The distributions were obtained by averaging the first $75 \mathrm{msec}$ of each TF representation of amplitude envelopes. Bottom panel, Averaged frequency distribution showing peaks around 20 and $60 \mathrm{~Hz}$. D, Top panel, An averaged TF representation of the 44 bursts in this experiment. The time scale is the same as in $B$. Note the pronounced peaks around 120 and $210 \mathrm{~Hz}$. Bottom panel, An averaged TF representation of the amplitude modulation.

$\mu \mathrm{M})$ completely blocked the sharp currents as well as the spontaneous network bursts ( $n=7$; data not shown).

We analyzed temporal patterning using Gabor-wavelet based $\mathrm{TF}$ analysis. Because of the highly time-variant frequency content of the current bursts, we found the TF analyses better suited than the conventional methods of frequency analysis, autocorrelograms and power spectra. In individual current bursts, both gamma- and high-frequency components could be identified
(Figs. $3 A, B, 4 A, B$ ), much as in single field potential bursts. The high-frequency components were in most recordings formed by small clusters of currents. To test the stability of these oscillations, we averaged TF representations within recordings. We consistently found peaks both in the gamma- and high-frequency bands (Figs. 3C,E, 4D). Neurons showing pronounced gamma oscillations (Fig. $3 A$ ) typically had a rather smooth, small amplitude distribution of higher frequencies and had a below average num- 


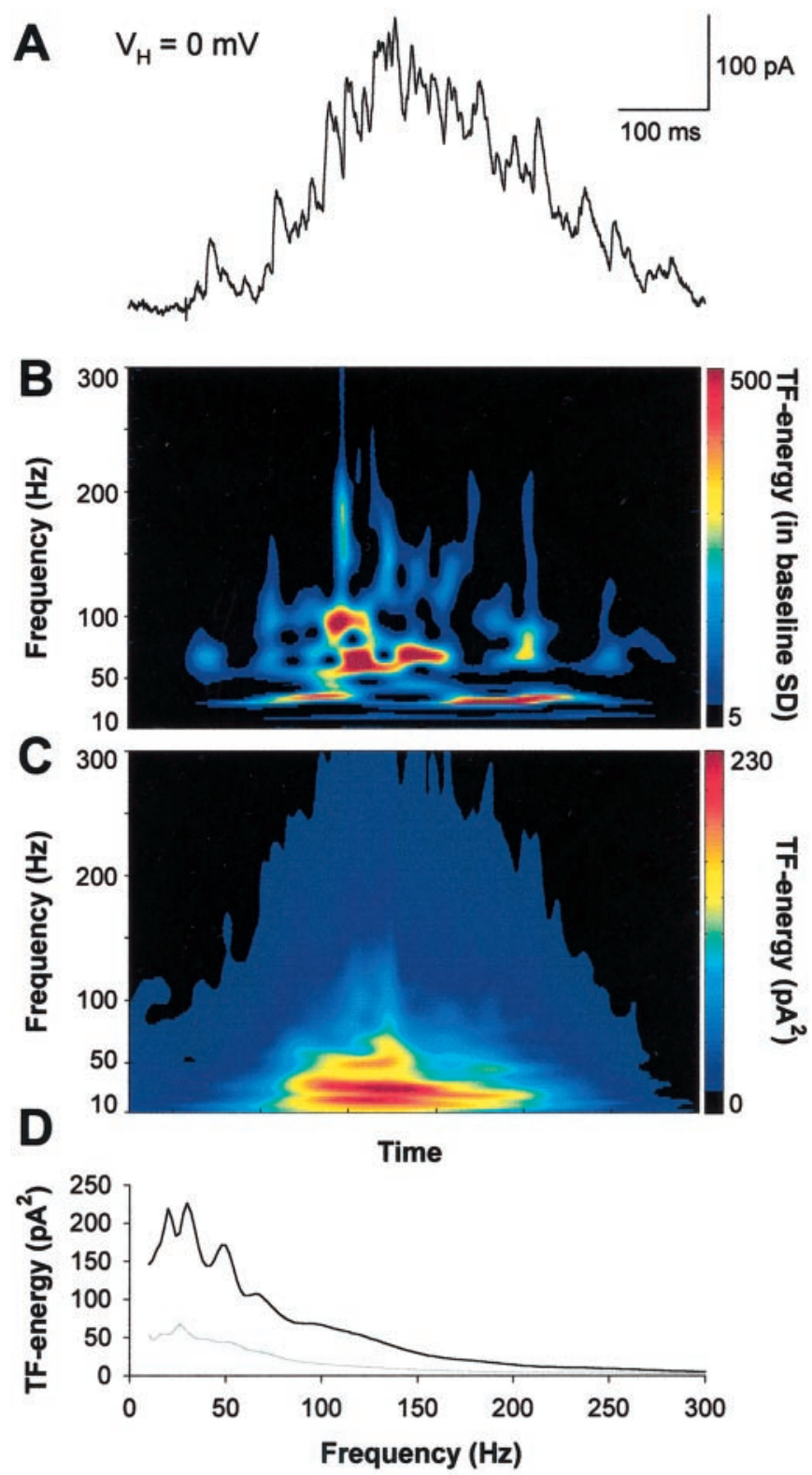

Figure 5. Interneuronal networks produce gamma- and high-frequency oscillations in immature hippocampus. $A$, A spontaneous event in a $\mathrm{P} 5$ pyramidal neuron (the same as in Fig. 3) whole-cell voltage-clamped near the AMPA-kainate reversal potential $(0 \mathrm{mV})$ demonstrating the GABAergic currents. $B$, A TF representation of the GABA currents in $A$. Note the series of brief high-frequency (here $100-200 \mathrm{~Hz}$ ) components over the prominent oscillation in the gamma-frequency band. $C$, An averaged TF representation of 101 bursts of GABAergic currents showing a major peak in the $20-40 \mathrm{~Hz}$ range. Note the similarity between the frequency contents of GABA and AMPA-kainate (Fig. 3) currents in the lower gamma-band of the averaged TF representations. $D$, Frequency distribution of the GABA currents computed from $C$ to allow for a comparison with Figure 3. Gray line indicates 3 SDs of preburst noise.

ber of AMPA-kainate current peaks. Conversely, neurons showing pronounced high-frequency oscillations (Fig. 4A) typically had only a minor gamma-frequency contribution and an above average number of AMPA-kainate current peaks. The correlation of the frequency distribution with the mean number of current peaks was highly significant on both gamma- and highfrequency bands (Fig. $3 F$ ). Similarly, also the mean interpeak interval was dependent on the number of AMPA-kainate current peaks ( $p<0.005$, ANOVA) (Fig. 3D).

The findings are thus consistent with the hypothesis that the pyramidal neurons participate in a high-frequency oscillation that is adequately reflected only in neurons receiving massively convergent projections from the pyramidal neurons. The frequency distributions were always broad, indicating large interburst variability. This is in line with the recent studies in the adult brain, where the gamma frequency network oscillations exhibit a large variability in their oscillation frequency and are prone to become averaged out when data are pooled (Gray and Di Prisco, 1997 and references therein; Brecht et al., 1999;).

\section{High-frequency population oscillation is modulated at gamma frequencies}

In field potential spike and AMPA-kainate current bursts, the brief high-frequency components appeared often to occur at gamma frequencies. Moreover, gamma frequency patterning was also imposed on the overall occurrence of field potential spikes. To quantify the possible rhythmic amplitude modulation of highfrequency oscillations, we averaged the amplitude envelope of the high-frequency band around the major peak or peaks in the original TF representations and then performed a second TF analysis to the amplitude envelope (Fig. 4A,B). We analyzed data from neurons receiving an above average number of AMPAkainate inputs and thereby showing more pronounced and continuous high-frequency oscillations $(n=3)$. Pronounced peaks in the gamma-frequency range were seen for the amplitude modulation of individual bursts (Fig. $4 C$ ) with exceptional signal-tonoise ratio; the peaks were typically two to three orders of magnitude greater than the preburst SD. In averaged amplitude modulation TF representations (Fig. 4D) broad peaks were found in both lower and upper gamma frequency bands.

The high-frequency pyramidal cell population oscillation was thus modulated at gamma frequencies. In neurons showing a large number of AMPA-kainate currents, the modulation appears as amplitude modulation of the high-frequency currents. Consequently, because of the modulation, the AMPA-kainate currents or the small current clusters appear at gamma frequencies in neurons receiving less projections from pyramidal cells.

\section{Gamma oscillations in immature interneuron networks}

During the field potential bursts, pyramidal neurons recorded at a distance of 100-200 $\mu \mathrm{m}$ from the extracellular electrode and voltage-clamped close to $0 \mathrm{mV}$ always displayed bursts of outward currents (Fig. 5A). The individual postsynaptic currents (PSCs) had a decay time-constant of $11 \pm 2 \mathrm{msec}(n=20$ cells $)$ and a reversal potential $\sim 20 \mathrm{mV}$ more positive than the $\mathrm{E}_{\mathrm{Cl} \text { - }}$ (Kaila, 1994), thereby implying a $\mathrm{GABA}_{\mathrm{A}}$ ergic origin (Hollrigel and Soltesz, 1997). Like AMPA-kainate currents, the GABA currents during the bursts displayed complex kinetics. TF-analyses of the individual barrages of GABAergic currents typically displayed pronounced peaks in the both lower and upper gamma-frequency bands as well as brief but prominent components at higher frequencies (Fig. 5B). Averaging the TF representations smoothed the upper gamma and high-frequency ranges and highlighted the lower gamma-frequency oscillations (Fig. 5C,D). Overall, the frequency distributions of GABAergic currents were very similar to the frequency distributions of AMPA-kainate currents. Whether the high-frequency components found in individual GABA current bursts reflect a more sustained high-frequency oscillation remains to be elucidated. 

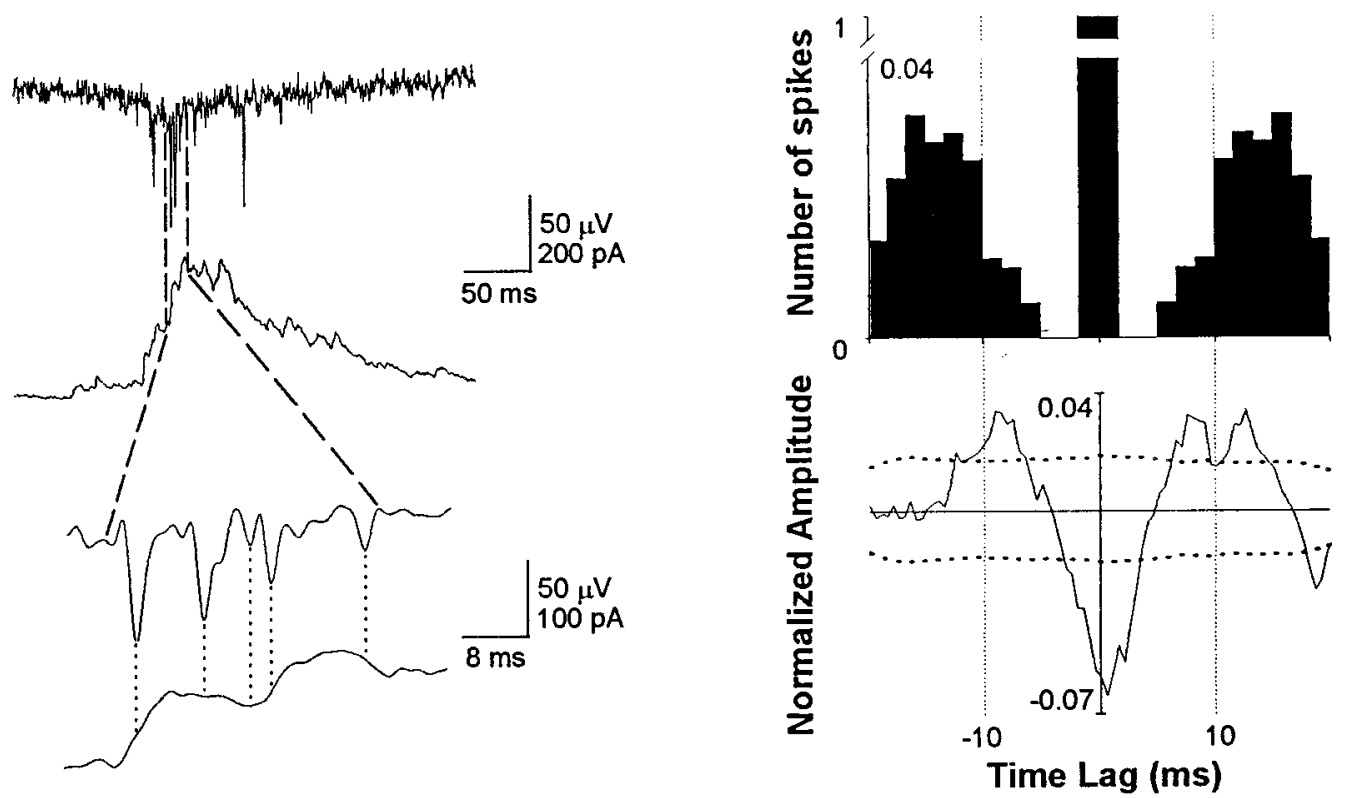

Figure 6. Field potential spikes are often found on ascending and descending slopes of GABAergic currents and only rarely on the current peaks. $A$, A barrage of GABAergic currents is seen in pyramidal neurons during the population events. The intracellular recording (bottom trace) is simultaneous with the field potential recording (top trace). The neuron was recorded at a distance of $100 \mu \mathrm{m}$ from the extracellular electrode and whole-cell voltage-clamped to $\sim 0 \mathrm{mV}$. B, Correlation of the field potential spikes and GABAergic currents. Top panel, Distribution of field potential spikes around the spike occurring at $t=0$. The values are pooled from six experiments and 805 spikes in 156 spontaneous events. Bottom panel, Mean GABAergic current around a field potential spike occurring at $t=0$, dotted lines indicate the SEM (see Materials and Methods for details). As the GABAergic currents are positive (outward), negative mean values indicate that field potential spikes are inversely correlated with the GABAergic currents.

\section{Role of GABA in pyramidal cell function during network oscillations}

Intrigued by the correspondence of $20-40 \mathrm{~Hz}$ oscillations in GABAergic currents and the modulation of high-frequency AMPA-kainate currents at the same frequencies, we investigated the role of GABAergic transmission further. Field potential spikes appeared often to occur on the rising or falling slopes of the GABAergic currents (Fig. 6A). To quantify the temporal relationship between the $\mathrm{GABA}_{\mathrm{A}}$ currents and population bursts, we averaged epochs of field potential spike-triggered GABAergic currents. The current amplitude was smallest at the time of the field potential spike (Fig. 6B; 804 averages recorded from six slices), indicating that there is an inverse correlation between the field potential spikes and the peaks of GABAergic currents.

To elucidate the firing pattern of single pyramidal neurons, we in some experiments acquired data in cell-attached configuration before entering into whole-cell recording. The spontaneous mean interspike interval was $7 \pm 4 \mathrm{sec}$. When the firing was associated with field potential bursts, neurons typically fired often only one or two spikes and occasionally bursts of up to five spikes (Fig. 7A). The firing rates in spike bursts were confined to frequencies $<150$ $\mathrm{Hz}$ and again mainly to the lower gamma band, $20-40 \mathrm{~Hz}$ (Fig. $7 A$, bottom panel; $n=5$ cells).

The firing patterns of pyramidal cells were dramatically affected by the $\mathrm{GABA}_{\mathrm{A}}$ antagonist bicuculline $(10 \mu \mathrm{M})$. The number of spikes per burst was increased to $10 \pm 1$ (range, 6-14; Fig. $7 B$ ), and the maximum frequency attained was shifted from gamma frequencies close to $200 \mathrm{~Hz}$ (Fig. 7B, bottom panel; $n=4$ cells). In field potential recordings, bicuculline increased the field potential spike amplitudes from $90 \pm 18 \mu \mathrm{V}$ to $220 \pm 64 \mu \mathrm{V}$ (Fig. $7 C ; p<0.01$, unpaired $t$ test, $n=5$ slices).

\section{DISCUSSION}

\section{Joint gamma- and high-frequency oscillations}

To the best of our knowledge, this is the first characterization of synaptically driven, joint gamma- $(20-100 \mathrm{~Hz})$ and highfrequency $(>100 \mathrm{~Hz})$ network oscillations in newborn brain. During the network oscillations, pyramidal neurons fired sparsely and mainly at lower gamma frequencies (see also Smith et al., 1990). The data, however, are consistent with the hypothesis that the firing was sharply synchronized into a high-frequency oscillation that was partially detected by field potential electrodes but properly reflected only in intracellular recordings from neurons receiving convergent projections from a large number pyramidal neurons. The high-frequency population oscillation was modulated at gamma frequencies to the extent that in most neurons the AMPA-kainate currents were seen predominantly at gamma frequencies with only subtle indications to the underlying highfrequency oscillation. The distribution of the high-frequency population discharge into gamma-frequency AMPA-kainate receptormediated excitation of target subpopulations may also reflect specific patterns of connectivity in the developing network.

Gamma- and high-frequency oscillations have not been found to occur simultaneously in an adult hippocampus and appear to be a unique feature of the developing cortical networks. Analogously to the gamma-modulated high-frequency oscillation, however, gamma oscillations in the adult hippocampus are correlated with and modulated by theta $(4-12 \mathrm{~Hz}$ ) oscillations (Soltesz and Dêschenes, 1993; Bragin et al., 1995). It has been suggested that the gamma oscillations nested in a theta cycle host a sequence of neural representations (Lisman and Idiart, 1995; Jensen and Lisman, 1996; Jensen et al., 1996). Similarly, during the high- 

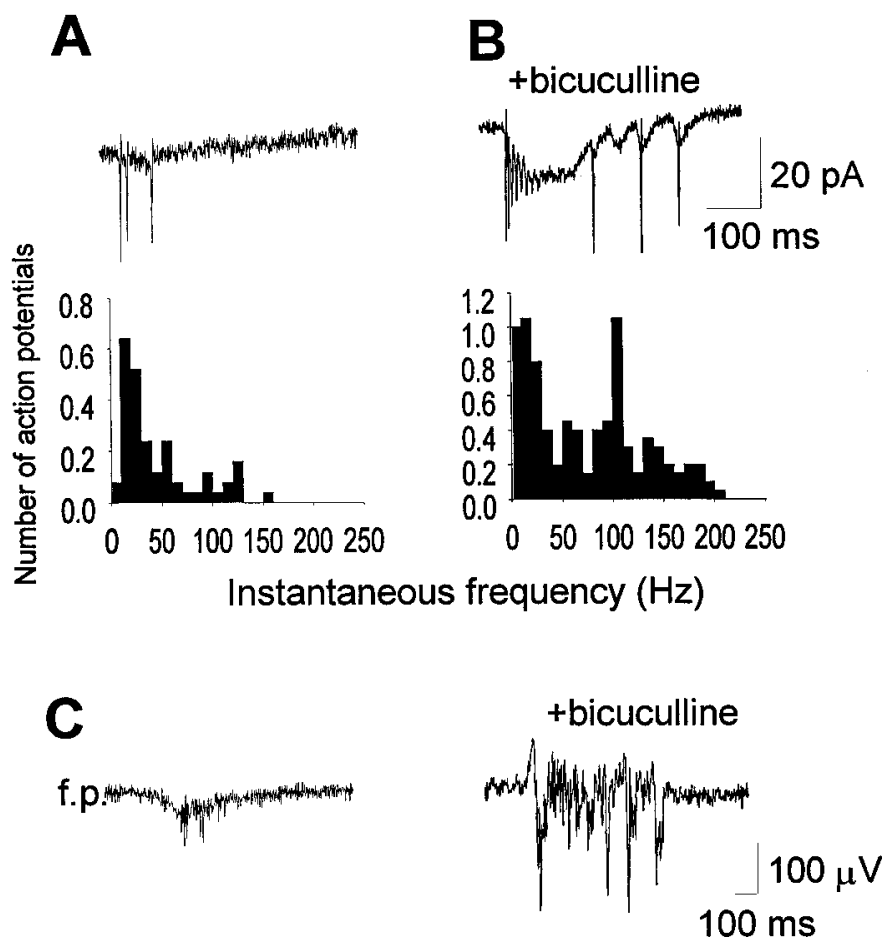

Figure 7. $\mathrm{GABA}_{\mathrm{A}}$ receptor-mediated transmission restrains the activity of both the individual pyramidal cells and the pyramidal cell population. $A$, A typical firing pattern of a pyramidal neuron recorded in a cellattached configuration. The sharp downward deflections are action potentials. $B$, Cell-attached recording of a spontaneous burst during a bath application of bicuculline methiodide $(10 \mu \mathrm{M})$. A, B, Bottom panels, Respective histograms of the action potential instantaneous frequencies in control (pooled from five cells and 25 spike bursts) and in bicuculline (pooled from four cells and 20 spontaneous events). The histograms are normalized with the number of events; the $y$-axis thus depicts the mean number of spikes (occurring during a single spontaneous event) in corresponding $10 \mathrm{~Hz}$ frequency bins along the $x$-axis. $C$, A field potential recording showing a population burst under control conditions and in the presence of bicuculline.

frequency oscillation within one gamma cycle, subpopulations of pyramidal neurons discharge sequentially.

\section{GABAergic transmission and gamma oscillations in the newborn hippocampus}

In rat hippocampal pyramidal neurons, GABAergic transmission has been reported to be depolarizing during the first postnatal week (Ben-Ari et al., 1989), and GABA has been suggested to provide the major excitatory drive to neonatal neurons together with the NMDA receptor-mediated transmission (c.f. Cherubini et al., 1991; Ben-Ari et al., 1997). Nevertheless, during the fast oscillations described here, AMPA-kainate receptor activation was responsible for the excitatory drive whereas the GABAergic transmission was clearly inhibitory. GABA currents were out-ofphase with the field potential spikes and restrained both the activity of individual pyramidal neurons as well as the operation of the pyramidal cell population. A slight depolarization by $\mathrm{GABA}_{\mathrm{A}} \mathrm{R}$ activation seen in neonatal neurons does not necessarily compromise functional inhibition (Qian and Sejnowski, 1990; Staley and Mody, 1992). Indeed, the inhibitory action of GABA seen here is based on nonhyperpolarizing, shunting-type inhibition (Lamsa et al., 2000).

In the adult hippocampus, the gamma oscillations may arise in networks of interneurons that entrain the firing of pyramidal cells by hyperpolarizing GABA $_{\mathrm{A}}$ ergic IPSPs (Lytton and Sejnowski, 1991; Cobb et al., 1995; Fisahn et al., 1998). Intriguingly, the GABAergic current peaks during the population burst occurred predominantly at gamma frequencies similarly to what has been found to follow a tetanic stimulation or glutamate injection in adults (Whittington et al., 1995). Computational models of interneuron networks have emphasized the need for synaptic hyperpolarization in the establishment of synchronous gamma rhythmicity (Wang and Buzsaki, 1996). Nevertheless, neonatal networks of interneurons may generate gamma oscillations during spontaneous bursts in the same manner as in adult hippocampus.

The population oscillation reached frequencies in the range of 300-400 Hz. It seems impossible for chemical synapses to synchronize neuronal activity at such high frequencies. It has recently been shown that in the absence of synaptic transmission, electrical coupling by gap junctions can also effectively synchronize the activity of pyramidal neurons in both neonate and adult hippocampus (Draguhn et al., 1998). Indeed, gap junction blockers have been shown to abolish the bursts of network activity in neonatal hippocampus (Strata et al., 1997). We thus propose that the pyramidal neurons, synchronized by gap junctions, form a high-frequency oscillation that is modulated at gamma, and possibly also at high frequencies, by the GABAergic interneurons.

\section{Implications for the activity-dependent wiring of the developing circuitry}

One of the putative functions of gamma rhythmicity in adults is long-range synchronization of neural activity, which in turn has been proposed to underlie feature and cognitive binding in distributed networks (Gray et al., 1989; König et al., 1995; Ritz and Sejnowski, 1997). It is tempting to speculate that the gamma- and high-frequency oscillations were to perform similar functions in the immature brain, albeit for different purposes. Indeed, we have recently demonstrated that the type of activity described here may be critical in the allocation of synaptic resources in the newborn rat hippocampus (Taira et al., 1998). Temporally correlated neural activity is critical in wiring the developing networks (Katz, 1993) and has been previously described to occur in tens of milliseconds or slower time scale (Garaschuk et al., 1998; Traub et al., 1998). Temporal precision has been shown to be essential in adult synaptic plasticity (Bell et al., 1997; Markram et al., 1997). Millisecond range synchronization during the fast network oscillations would thus allow for sharper coincidence detection and could be used for more precise shaping of the developing synaptic contacts. This scheme may add to the recapitulative mechanisms used during developmental synapse formation and synaptic plasticity in the adults.

\section{Conclusions}

We show here that a sequential synchronized discharge of distributed pyramidal cell groups gives rise to a high-frequency population oscillation in the rat hippocampus during the first postnatal days. Temporal patterns in the bursts represent a novel form of correlated activity that may sculpt connectivity within the developing synaptic circuitry.

\section{REFERENCES}

Bell CC, Han VZ, Suguwara Y, Grant K (1997) Synaptic plasticity in a cerebellum-like structure depends on temporal order. Nature 387:278-281.

Ben-Ari Y, Cherubini E, Corradetti R, Gaiarsa JL (1989) Giant synaptic potentials in immature rat CA3 hippocampal neurones. J Physiol (Lond) 416:303-325. 
Ben-Ari Y, Khazipov R, Leinekugel X, Caillard O, Gaiarsa J-L (1997) $\mathrm{GABA}_{\mathrm{A}}, \mathrm{NMDA}$ and AMPA receptors: a developmentally regulated "ménage à trois". Trends Neurosci 20:523-529.

Bragin A, Jando G, Nadasdy Z, Hetke J, Wise K, Buszaki G (1995) Gamma $(40-100 \mathrm{~Hz})$ oscillation in the hippocampus of the behaving rat. J Neurosci 15:47-60.

Brecht M, Singer W, Engel AK (1999) Patterns of synchronization in the superior colliculus of anaesthetized cats. J Neurosci 19:3467-3579.

Cherubini E, Gaiarsa J-L, Ben-Ari Y (1991) GABA: an excitatory transmitter in early postnatal life. Trends Neurosci 14:515-519.

Cobb SR, Buhl EH, Halasy K, Paulsen O, Somogyi P (1995) Synchronization of neuronal activity in hippocampus by individual GABAergic interneurons. Nature 378:78-78.

Draguhn A, Traub RD, Schmitz D, Jefferys JGR (1998) Electrical coupling underlies high-frequency oscillations in the hippocampus in vitro. Nature 394:189-192.

Durand GM, Kovalchuk Y, Konnerth A (1996) Long-term potentiation and functional synapse induction in developing hippocampus. Nature 381:71-75.

Fisahn A, Pike FG, Buhl E, Paulsen O (1998) Cholinergic induction of network oscillations at $40 \mathrm{~Hz}$ in the hippocampus in vitro. Nature 394:186-189.

Garaschuk O, Hanse E, Konnerth A (1998) Developmental profile and synaptic origin of early network oscillations in the CA1 region of rat neonatal hippocampus. J Physiol (Lond) 507.1:219-236.

Gray CM, Di Prisco GV (1997) Stimulus-dependent neuronal oscillations and local synchronization in striate cortex of the alert cat. J Neurosci 17:3239-3253.

Gray CM, König P, Engel AK, Singer W (1989) Oscillatory responses in cat visual cortex exhibit inter-columnar synchronization which reflects global stimulus properties. Nature 338:334-337.

Hestrin S, Nicoll RA, Perkel DJ, Sah P (1990) Analysis of excitatory synaptic action in pyramidal cells using whole-cell recording from rat hippocampal slices. J Physiol (Lond) 422:203-25.

Hollrigel GS, Soltesz I (1997) Slow kinetics of miniature IPSCs during early postnatal development in granule cells of the dentate gyrus. J Neurosci 17:5119-5128.

Jensen O, Lisman JE (1996) Hippocampal CA3 region predicts memory sequences: accounting for the phase precession of place cells. Learn Mem 3:279-287.

Jensen O, Idiart MAP, Lisman JE (1996) Physiologically realistic formation of autoassociative memory in networks with theta/gamma oscillations: role of fast NMDA channels. Learn Mem 3:243-256.

Kaila K (1994) Ionic basis of $\mathrm{GABA}_{\mathrm{A}}$ receptor channel function in the nervous system. Prog Neurobiol 42:489-537.

Katz LC (1993) Coordinate activity in retinal and cortical development. Curr Opin Neurobiol 3:93-99.

Katz LC, Shatz CJ (1996) Synaptic activity and the construction of cortical circuits. Science 274:1133-1138.

König P, Engel AK, Singer W (1995) Relation between oscillatory activity and long-range synchronization in cat visual cortex. Proc Natl Acad Sci USA 92:290-294.

Lamsa K, Palva JM, Ruusuvuori E, Kaila K, Taira T (2000) Synaptic $\mathrm{GABA}_{\mathrm{A}}$ activation inhibits AMPA/kainate receptor-mediated bursting in the newborn ( $\mathrm{P} 0-\mathrm{P} 2)$ rat hippocampus. J Neurophysiol, in press.

Laurent G (1997) Olfactory processing: maps, time and codes. Curr Opinion Neurobiol 7:547-553.

Lisman JE, Idiart MAP (1995) Storage of $7+/-2$ short-term memories in oscillatory subcycles. Science 267:1512-1514.
Leinekugel X, Medina I, Khalilov I, Ben-Ari Y, Khazipov R (1997) $\mathrm{Ca}^{2+}$ oscillations mediated by the synergistic excitatory actions of $\mathrm{GABA}_{\mathrm{A}}$ and NMDA receptors in the neonatal hippocampus. Neuron $18: 243-25$.

Luhmann HJ, Prince DA (1991) Postnatal maturation of the GABAergic system in the rat. J Neurophysiol 65:247-263.

Lytton WW, Sejnowski TJ (1991) Simulations of cortical pyramidal neurons synchronized by inhibitory interneurons. J Neurophysiol 66:1059-1079.

Markram H, Lübke J, Frotscher M, Sakmann B (1997) Regulation of synaptic efficacy by coincidence of postsynaptic APs and EPSPs. Science 275:213-215.

O'Donovan MJ (1999) The origin of spontaneous activity in developing networks of the vertebrate nervous system. Curr Opin Neurobiol 9:94-104.

Qian N, Sejnowski TJ (1990) When is an inhibitory synapse effective? Proc Natl Acad Sci USA 87: 8145-8149.

Ritz R, Sejnowski TJ (1997) Synchronous oscillatory activity in sensory systems: new vistas on mechanisms. Curr Opin Neurobiol 7:536-546.

Rivera C, Voipio J, Payne JA, Ruusuvuori E, Lahtinen H, Lamsa K, Pirvola U, Saarma M, Kaila K (1999) A K-Cl cotransporter KCC2 renders GABA hyperpolarising during neuronal maturation. Nature 397:251-255.

Soltesz I, Deschênes M (1993) Low- and high-frequency membrane potential oscillations during theta activity in CA1 and CA3 pyramidal neurons of the rat hippocampus under ketamine-xylazine anesthesia. J Neurophysiol 70:97-115.

Sinkkonen J, Tiitinen H, Näätänen R (1995) Gabor filters: an informative way for analyzing event-related brain activity. J Neurosci Methods 56:99-104.

Smith JC, Greer JJ, Liu GS, Feldman JL (1990) Neural mechanisms generating respiratory pattern in mammalian brainstem-spinal cord in vitro. 1. Spatiotemporal patterns of motor and medullary neuron activity. J Neurophysiol 64:1149-1169.

Staley KJ, Mody I (1992) Shunting of excitatory input to dentate gyrus granule cells by a depolarising $\mathrm{GABA}_{\mathrm{A}}$ receptor-mediated postsynaptic conductance. J Neurophysiol 68:197-212.

Strata F, Atzori M, Molnar M, Ugolini G, Tempia F, Cherubini E (1997) A pacemaker current in dye-coupled hilar interneurons contributes to the generation of giant GABAergic potentials in developing hippocampus. J Neurosci 17:1435-1446.

Taira T, Lauri SE, Lamsa K, Rauvala H, Kaila K (1998) Network activity-dependent synapse elimination in neonatal rat hippocampus. Soc Neurosci Abstr 24:415.10.

Traub RD, Spruston N, Soltesz I, Konnerth A, Whittington MA, Jefferys JGR (1998) Gamma-frequency oscillations: A neuronal population phenomenon, regulated by synaptic and intrinsic cellular processes, and inducing synaptic plasticity. Prog Neurobiol 55:563-567.

Wang X-J, Buzsaki G (1996) Gamma oscillation by synaptic inhibition in a hippocampal interneuronal network model. J Neurosci 16:6402-6413.

Whittington MA, Traub RD, Jefferys JGR (1995) Synchronized oscillations in interneuron networks driven by metabotropic glutamate receptor activation. Nature 373:612-615.

Ylinen A, Bragin A, Nadasdy Z, Jando G, Szabo I, Sik A, Buszaki G (1995) Sharp wave-associated high-frequency oscillation $(200 \mathrm{~Hz})$ in the intact hippocampus: network and intracellular mechanisms. J Neurosci 15:30-46. 